\title{
DIAGNÓSTICO DE USO E CONSERVAÇÃO DO SOLO POR AGRICULTORES NO NORDESTE BRASILEIRO
}

Lucas de Sousa Oliveira ${ }^{1}$, Alfredo Mendonça de Sousa ${ }^{3}$, José Israel Pinheiro², Danilo Batista Nogueira ${ }^{3}$, Mateus Guimarães da Silva ${ }^{2}$

${ }^{1}$ Bacharel em Agronomia, Doutorando em Ciências do Solo/Universidade Federal do

Ceará (UFC), Fortaleza-CE, Brasil. E-mail: lucasdesousa@alu.ufc.br

${ }^{2}$ Bacharel em Agronomia, Doutorando em Ciências do Solo/Universidade Federal do Ceará (UFC), Fortaleza-CE, Brasil.

${ }^{3}$ Bacharel em Agronomia, Doutorando em Engenharia Agrícola/Universidade Federal do Ceará (UFC), Fortaleza-CE, Brasil.

Recebido em: 15/05/2020 - Aprovado em: 15/06/2020 - Publicado em: 30/06/2020 DOI: 10.18677/EnciBio_2020B24

\section{RESUMO}

A agricultura tradicional priorizou um modelo com base no uso intensivo da terra, o que gerou inúmeros problemas ambientais e econômicos, com destaque para a degradação dos solos. Tais sistemas agrícolas, cujo processo de produção está baseado apenas na maximização da produção e no lucro, não consideram a capacidade de uso das terras, o que resulta, em muitas ocasiões, em diminuição da qualidade do solo. Desse modo, objetivou-se diagnosticar as práticas de uso da terra empregadas pelos agricultores de um município da região Nordeste do Brasil, identificando as vulnerabilidades e a viabilidade de adoção de práticas conservacionistas. Para tanto, foi utilizada a metodologia do diagnóstico participativo, que consiste em visitas in situ. Para execução da metodologia citada, foi elaborado/aplicado um questionário com as 12 famílias do povoado em estudo (povoado Canafista). A maioria das práticas adotadas no povoado é inadequada, gerando degradação de extensas áreas cultivadas. Existe a necessidade de orientação técnica para adoção de práticas conservacionistas e maior facilidade de acesso ao crédito rural para implementação do uso sustentável da terra.

PALAVRAS-CHAVE: agricultura familiar, desenvolvimento sustentável, manejo do solo.

\section{DIAGNOSIS OF SOIL USE AND CONSERVATION BY FARMERS IN NORTHEAST BRAZIL}

\begin{abstract}
Traditional agriculture prioritized a model based on intensive land use, which generated numerous environmental and economic problems, with emphasis on soil degradation. Such agricultural systems, whose production process is based solely on maximizing production and profit, do not consider the land use capacity, which often results in a decrease in soil quality. Thus, the objective was to diagnose the land use practices employed by farmers in a municipality in the Northeast region of Brazil, identifying the vulnerabilities and the feasibility of adopting conservationist practices. For this, the participatory diagnosis methodology was used, which consists of on-site
\end{abstract}


visits. In order to carry out the aforementioned methodology, a questionnaire was prepared / applied with the 12 families of the village under study (Canafista village). Most of the practices adopted in the village are inadequate, causing degradation of extensive cultivated areas. There is a need for technical guidance for the adoption of conservationist practices and easier access to rural credit for the implementation of sustainable land use.

KEY WORDS: family farming, sustainable development, soil management.

\section{INTRODUÇÃO}

A agricultura familiar tem dinâmica e características bem peculiares. Nela, a gestão da propriedade é compartilhada e a atividade produtiva, seja agricultura e/ou pecuária, é a principal fonte geradora de renda. Conforme dados do Instituto Brasileiro de Geografia e Estatística (2017), ressalta-se a importância dessa modalidade de agricultura para o país; segundo os pesquisadores, são classificados como agricultura familiar, cerca de 3,9 milhões de estabelecimentos, sendo estes distribuídos por cerca de 80 milhões de hectares.

No tocante à região Nordeste, Vidal e Santos (2016) chamaram atenção que essa região possui mais da metade dos estabelecimentos rurais do país. Almeida et al. (2017) em seu estudo salientam que nessa região há predomínio de sistemas de produção desenvolvidos em pequenas áreas e, normalmente, de baixa eficiência.

A principal atividade econômica em muitos povoados/assentamentos na região Nordeste é a agropecuária. Nesse aspecto, destaca-se que a maioria dessas regiões apresentam solos de baixa fertilidade, baixa infraestrutura e com condições climáticas que são pouco favoráveis para a maioria das formas de exploração/uso pelos cultivos.

Segundo Oliveira Filho et al. (2019) a agricultura praticada nessas regiões envolve técnicas como o desmatamento e as queimadas que levam à degradação de extensas áreas. Essas práticas, geralmente associadas à produção animal (superpastejo), favorecem a degradação do ambiente e a redução do potencial produtivo das terras (ZERAATPISHEH et al., 2020).

A remoção da cobertura vegetal provoca mudanças na dinâmica de funcionamento do solo, podendo reduzir seu potencial de produção por perdas de matéria orgânica e nutrientes levando a um cenário de degradação do solo (MARTíN et al., 2019). Pendrill et al. (2019) sugeriram que a agricultura e a pecuária são atividades relacionadas com o uso dos solos, e mudanças decorrentes de práticas de manejo inadequadas, podem levar a um cenário de rápido declínio nos estoques de carbono e nutrientes, colaborando para o aumento das emissões de gases do efeito estufa (GEE) e desertificação de extensas áreas (ARMENTERAS et al., 2017).

Diante do exposto, o objetivo do estudo foi diagnosticar as práticas de uso da terra empregadas pelos agricultores, identificando as vulnerabilidades e a viabilidade de adoção de práticas conservacionistas.

\section{MATERIAL E MÉTODOS}

O estudo foi realizado no Povoado Canafista, localizado a 2,5 km da sede do município de Graça Aranha, Maranhão, cujas coordenadas geográficas são 05ำ 24'36" de latitude Sul e 44 20'02" de longitude Oeste. O clima da região é classificado como Aw, conforme Köppen, apresentando temperatura média anual de 26,6 ${ }^{\circ} \mathrm{C}$, período chuvoso concentrado nos meses de dezembro a abril com pluviosidade anual média de $1224 \mathrm{~mm}$ (Instituto Nacional de Pesquisas Espaciais, 2017). 
A principal fonte de renda dos agricultores do povoado é a pecuária, sendo cultivado, principalmente, pastagem para criação de animais (bovinos e ovinos). Além da criação de animais, a agricultura no povoado é baseada em pequenos cultivos de milho, feijão e mandioca, cultivados em sistema de sequeiro para subsistência. A área total das propriedades do povoado é de 632 ha.

Consta na Figura 1 as principais formas de uso do solo pelos agricultores do povoado Canafista, Graça Aranha, Maranhão.

FIGURA 1. Principais usos do solo no povoado Canafista, Graça Aranha, Maranhão. A: Criação de bovinos de corte; B: Área desmatada para plantio de feijão verde; C: Plantio de mandioca; D: Área de consórcio milho/mandioca/abóbora/melancia.

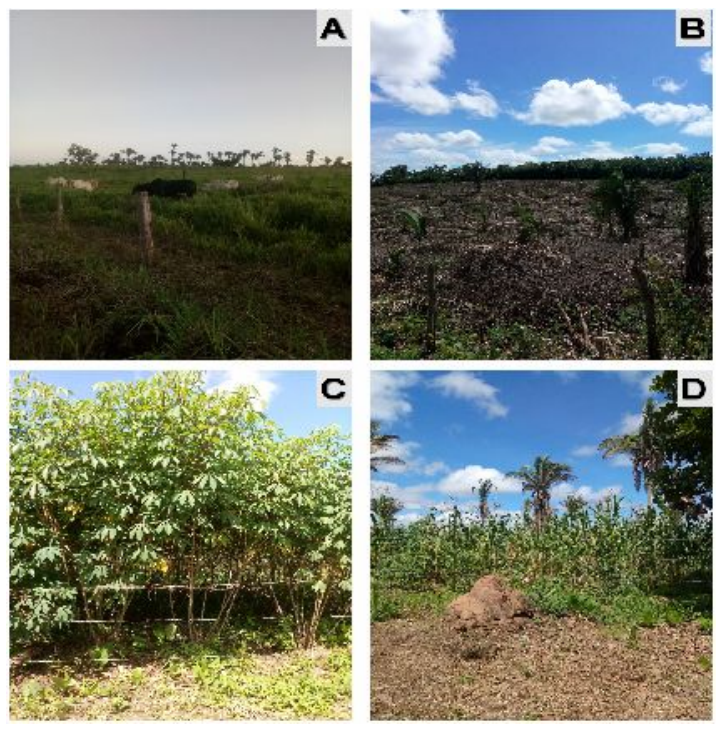

Fonte: Autores.

Na condução do estudo foi utilizada a metodologia do diagnóstico participativo, que consiste de visitas in situ. Para isso, foi elaborado/aplicado um questionário com as 12 famílias do povoado. Na ocasião das visitas domiciliares foi explanado o objetivo do estudo, de modo a dar ciência aos agricultores da importância do trabalho para a comunidade.

As perguntas de partida para a elaboração do questionário tiveram como base a percepção dos agricultores/agricultoras referentes à importância do manejo sustentável do solo, principais cultivos, mecanização, assistência técnica e das práticas utilizadas para manter o solo produtivo.

De posse das informações, os dados foram analisados e apresentados na forma de gráficos elaborados com auxílio do software SigmaPlot V.14.

\section{RESULTADOS E DISCUSSÃo}

A intervenção do homem no ambiente tem agravado de maneira expressiva a degradação dos solos pelo mundo. As práticas agrícolas inadequadas ou sem planejamento e a pecuária extensiva são fatores que intensificam a problemática, sobretudo em um espaço ambientalmente frágil (LEMMA et al., 2017). Com o desmatamento indiscriminado, queimadas, secas prolongadas, pressão demográfica e aumento da exploração agrícola, há diminuição da fertilidade dos solos dessas regiões. 
No que tange às principais formas de preparo do solo entre os agricultores, observa-se (FIGURA 2) que na maior parte das propriedades avaliadas no estudo o desmatamento da vegetação nativa associado ao uso do fogo ainda é bem recorrente.

FIGURA 2. Principais formas de preparo de solo entre os agricultores do Povoado Canafista, Graça Aranha, Maranhão.

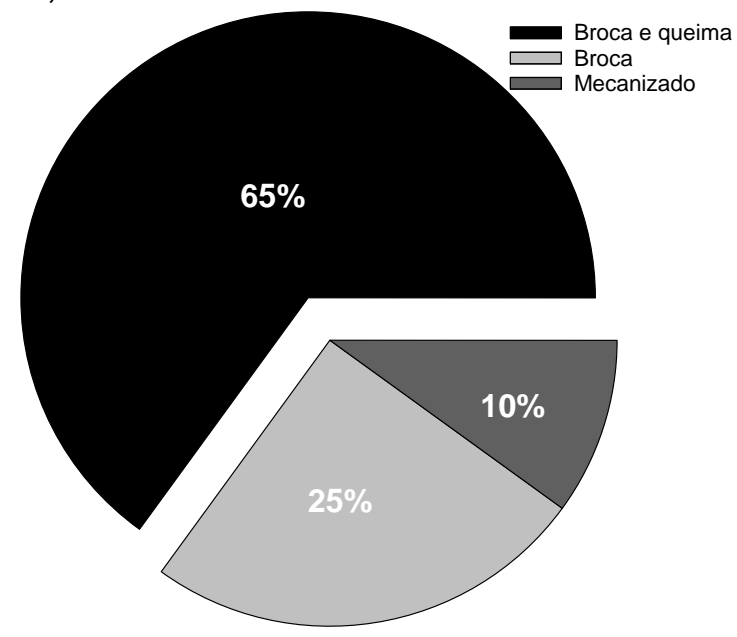

Fonte: Autores.

O uso do fogo em áreas de exploração agrícola pode ser considerado uma grave ameaça para a conservação da biodiversidade dos ambientes e manutenção dos processos ecológicos. Como consequências diretas do uso do fogo podemos citar, as perdas nos estoques de carbono, alterações no ciclo hidrológico e de nutrientes, aumento dos processos erosivos e o assoreamento da maioria das fontes hídricas (açudes, principalmente) (ROBICHAUD et al., 2017).

Segundo Robichaud et al. (2017) o uso do fogo como forma de manejar os solos agrícolas tem causado danos severos ao ambiente, comprometendo a qualidade e a manutenção da fauna e flora e impactando de forma negativa o desenvolvimento socioeconômico local. Nesse sentido, muitos estudos têm chamado a atenção para a ameaça do uso indiscriminado das queimadas no preparo das áreas de cultivo, indicando caminhos/soluções para investimentos na conservação dessas áreas (STAVI et al., 2016). Destaca-se que a falta de tais informações, ou o incipiente acesso a estas, impulsiona o aumento da prática de queimadas e o avanço nos processos de desmatamentos e degradação ambiental dessas áreas.

No estudo realizado no povoado observou-se que os cultivos agrícolas e as pastagens, na maioria das propriedades, são formados após a retirada da vegetação nativa e/ou secundária pelo uso do fogo em áreas declivosas sem respeitar a aptidão e uso e sem o manejo adequado dos solos, o que tem intensificado os processos erosivos, culminando com a diminuição da fertilidade dos solos, conforme pode-se observar na figura 1, apresentada anteriormente.

No tocante ao tipo de cultivo utilizado pelos agricultores observa-se (FIGURA 3) que o monocultivo ocupa a maior extensão territorial do povoado. Sabe-se que a rotação dos cultivos desempenha papel chave na manutenção da fertilidade dos solos, uma vez que, a ausência de diversidade de plantas em sistemas de cultivo agrícolas é fator chave no processo de degradação do solo. 
FIGURA 3. Principais sistemas de cultivo utilizados pelos agricultores no Povoado Canafista, Graça Aranha, Maranhão.

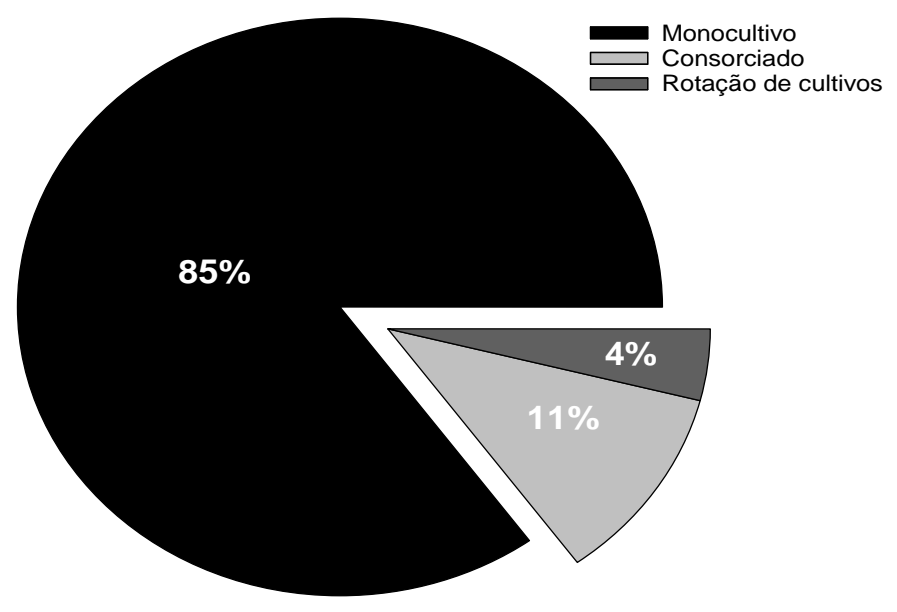

Fonte: Autores.

Estudos de Hussain et al. (2019) e Martín et al. (2019) constataram perdas de matéria orgânica do solo em áreas sob monocultivos. Logo, práticas conservacionistas que priorizem a manutenção de resíduos na cobertura do solo são indicadas quando se busca reduzir estas perdas, mantendo os teores de carbono orgânico e, em alguns casos, aumentando-os.

Conforme mencionado anteriormente, a pastagem bem manejada é agente importante na manutenção da matéria orgânica do solo. Viglizzo et al. (2019) salientaram que a maior contribuição das pastagens como promotoras do acúmulo de carbono orgânico no solo advém de suas raízes abundantes que exploram o perfil do solo distribuindo esse carbono em profundidade.

No que se refere a utilização de fertilizantes, o estudo indicou que apenas $8 \%$ do total de agricultores fazem uso de tal insumo, conforme exposto na figura 4.

FIGURA 4. Principais medidas tomadas pelos agricultores para manter a terra produtiva no Povoado Canafista, Graça Aranha, Maranhão.

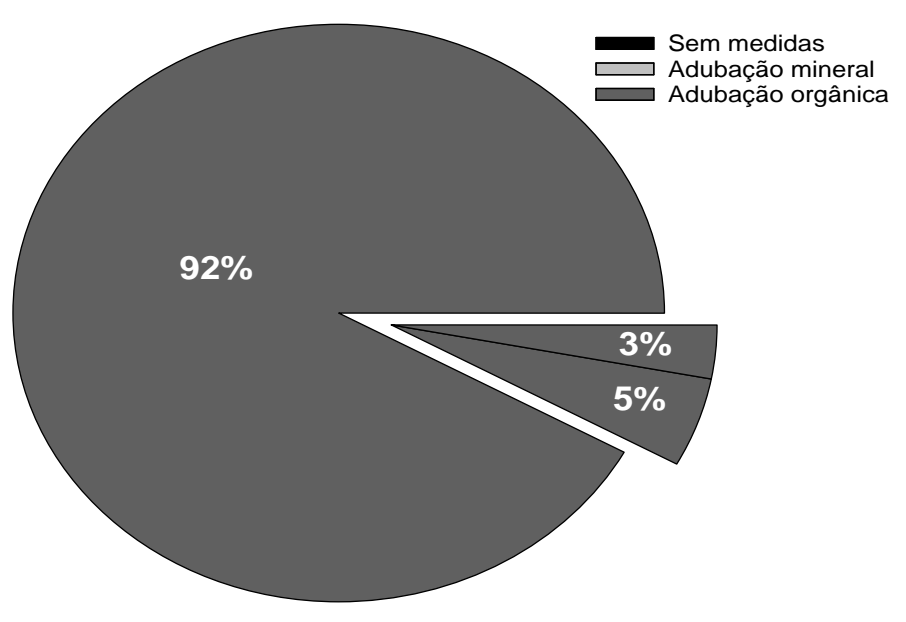

Fonte: Autores. 
O uso do solo, sem a adoção de medidas de produção sustentáveis, contribui para o processo de degradação física, química e biológica, resultando em redução da produtividade das culturas exploradas, cada vez mais acentuada com o contínuo manejo inadequado do solo (SILVA et al., 2015).

Os fertilizantes minerais utilizados pelos agricultores são de alto custo financeiro, o que ficou evidente na porcentagem de uso dessas fontes pelos agricultores do povoado (FIGURA 4). Outro detalhe que foi observado é que os agricultores que fazem uso de fontes minerais na suplementação dos cultivos, realizam tais aplicações sem conhecimento e/ou orientação técnica sobre as quantidades requeridas pelas plantas. Menezes et al. (2016) salientam que o uso excessivo de fertilizantes, minerais e/ou orgânicos, pode contribuir para contaminação do solo e de cursos d'água, acidificação do solo, além de emissões de gases do efeito estufa.

O uso da adubação orgânica como fonte de nutrientes é justificado pela necessidade de reciclagem desses materiais, uma vez que, uma considerável parte dos resíduos produzidos, até mesmo na própria propriedade, é de origem orgânica. Uma vez conhecida a composição mineral dos resíduos pode-se indicar seu uso no fornecimento de nutrientes essenciais para as plantas, podendo em alguns casos, substituir toda a adubação mineral.

É importante destacar, todavia, que o uso de adubação orgânica deve ser feito de forma criteriosa para que não se adicione ao solo quantidade maior que a requerida pelas culturas, evitando-se, desta forma, acúmulo de nutrientes no solo, conforme salientam Abreu Junior et al. (2002).

Observou-se a falta de orientação técnica para os agricultores no manejo/uso adequado do solo (FIGURA 5).

FIGURA 5. Principal fonte de orientação técnica acessível aos agricultores no Povoado Canafista, Graça Aranha, Maranhão.

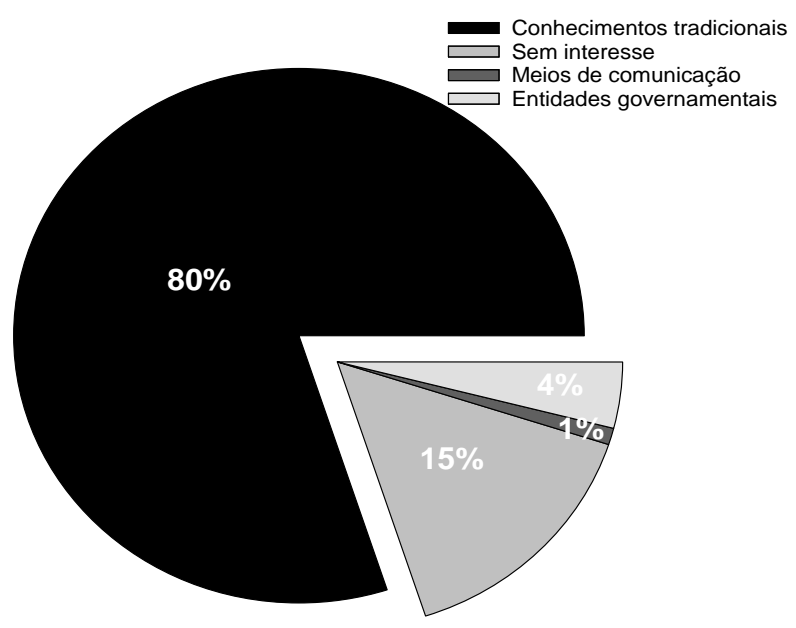

Fonte: Autores.

Mesmo com a modernização da agricultura, a agricultura familiar ficou à margem dos benefícios oferecidos pela politica agrícola, uma vez que as inciativas privilegiaram os setores mais capitalizados e a produção de commodities voltadas para o mercado internacional. Este cenário justifica o fato de apenas $4 \%$ dos agricultores ouvidos na pesquisa serem beneficiados com politicas governamentais, 
destacando-se também a falta de interesse (15\%) em obter informações de técnicos/extensionistas para o uso sustentável da terra (FIGURA 5).

Falta de assistência técnica e crédito gerou dificuldades para 0 desenvolvimento da agricultura familiar que tem longa tradição $(80 \%$ dos entrevistados usam como principal fonte de orientação o conhecimento tradicional). No entanto, observa-se que essa realidade vem sendo modificada, maximizando possibilidades pelos inúmeros debates que abrangem a importância desse segmento para a geração de renda e desenvolvimento do país.

Kassam et al., (2014) salientaram o fato de que instituições que gerem conhecimento devem alinhar-se cada vez mais em pesquisas, ensino e extensão, de modo a fomentar não somente a adoção como a difusão de sistemas agrícolas conservacionistas.

Ao analisar os riscos associados à degradação da terra observa-se que $85 \%$ dos produtores não estão preocupados com a adoção do manejo correto do solo (FIGURA 6).

FIGURA 6. Medidas para reduzir os riscos associados à degradação da terra no Povoado Canafista, Graça Aranha, Maranhão.

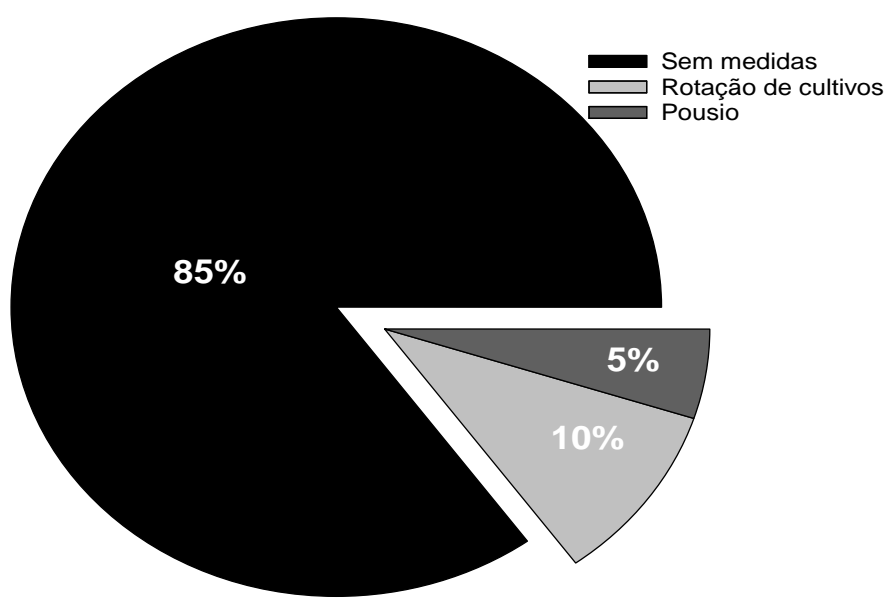

Fonte: Autores.

Segundo Oliveira Filho et al. (2019) o desmatamento, somado ao cultivo intensivo sem a adoção de práticas de manejo conservacionistas, resulta em declínio da fertilidade dos solos. A redução na fertilidade dos solos em áreas exploradas por agricultores familiares tem se agravado com os anos, colocando-se em pauta a capacidade de uso da terra para a manutenção das atividades produtivas.

Ressalta-se que a maioria dos agricultores entrevistados não tem informações suficientes sobre a importância de manejar corretamente a terra. Essa expressiva porcentagem (85\%) é justificada por fatores como baixa escolaridade, menor nível de eficiência técnica, dificuldades de acesso ao crédito e a assistência técnica constante.

Para adoção de uma agricultura sustentável há exigência de maior conhecimento, uma vez que o sucesso da adoção de práticas conservacionistas dependerá em grande parte da gestão feita pelos agricultores em suas propriedades, da renda disponível e de exemplos de que tais medidas são fundamentais para manutenção da função ecológica e agrícola dos solos. 


\section{CONCLUSÕES}

A maioria das práticas adotadas no Povoado Canafista são inadequadas, gerando degradação de extensas áreas cultivadas.

Existe a necessidade de orientação técnica para adoção de práticas conservacionistas e maior facilidade de acesso ao crédito rural para implementação do uso sustentável da terra.

\section{REFERÊNCIAS}

ABREU JUNIOR, C. H.; MURAOKA, T.; OLIVEIRA, F. C. Carbono, nitrogênio, fosforo e enxofre em solos tratados com composto de lixo urbano. Revista Brasileira de Ciência do Solo, v. 26, p. 769-780, 2002. DOI: doi.org/10.1590/S0100-06832002000300022.

ALMEIDA, J. A.; SANTOS, A. S.; NASCIMENTO, M. A. O.; OLIVEIRA, J. V. C.; SILVA, D. G.; MENDES-NETO, R. S. Fatores associados ao risco de insegurança alimentar e nutricional em famílias de assentamentos rurais. Ciência e Saúde Coletiva, v. 22, n. 2, p. 479-488, 2017. DOI: doi.org/10.1590/141381232017222.27102015.

ARMENTERAS, D.; ESPELTA, J. M.; RODRíGUEZ, N.; RETANA, J. Deforestation dynamics and drivers in different forest types in Latin America: Three decades of studies (1980-2010). Global Environmental Change, v. 46, p. 139-147, 2017. DOI: doi.org/10.1016/j.gloenvcha.2017.09.002.

HUSSAIN, S.; SHARMA, V.; ARYA, V. M.; SHARMA, K. R.; RAO, C. S. Total organic and inorganic carbon in soils under different land use/land cover systems in the foothill Himalayas. Catena, v. 182, n. 104104, 2019. DOI: doi.org/10.1016/j.catena.2019.104104.

Instituto Brasileiro de Geografia e Estatística. Senso Agropecuário 2017. Disponível em: https://www.ibge.gov.br/estatisticas/economicas/agricultura-epecuaria.html.

Instituto Nacional de Pesquisas Espaciais 2017. Disponível em: https://www.cptec.inpe.br.

KASSAM, A.; DERPSCH, R.; FRIEDRICH, T. Global achievements in soil and water conservation: The case of Conservation Agriculture. International Soil and Water Conservation Research, v. 2, n. 1, p. 5-13, 2014. DOI: doi.org/10.1016/S20956339(15)30009-5.

LEMMA, B.; KEBEDE, F.; MESFIN, S.; FITIWY, I.; ABRAHA, Z.; NORGROVE, L. Quantifying annual soil and nutrient lost by rill erosion in continuously used semiarid farmlands. Environment Earth Science, v.76, p. 190-198, 2017. DOI: doi.org/10.1007/s12665-017-6506-z.

MARTíN, J. A. R.; ÁlVARO-FUENTES, J.; GABRIEL, J. L.; GUITIÉRREZ, C.; NANOS, N.; ESCUER, M.; RAMOS-MIRAS, J. J.; GIL, C.; MARTÍN-LAMMERDING, D.; BOLUDA, R. Soil organic stock on the Majorca Island: Temporal change in agricultural soil over the last 10 years. Catena, v. 181, n. 104087, 2019. DOI: doi.org/10.1016/j.catena.2019.104087. 
MENEZES, J. P. C.; BITTENCOURT, R. P.; FARIAS, M. S.; BELLO, I. P.; FIA, R.; OLIVEIRA, L. F. C. Relação entre padrões de uso e ocupação do solo e qualidade da água em uma bacia hidrográfica urbana. Engenharia Sanitária e Ambiental, v. 2, n. 3, p. 519-534, 2016. DOI: doi.org/10.1590/S1413-41522016145405.

OLIVEIRA FILHO, J. S.; VEIRA, J. N.; SILVA, E. M. R.; OLIVEIRA, J. G. B.; PEREIRA, M. G.; BRASILEIRO, F. G. Assessing the effects of 17 years of grazing exclusion in degraded semi-arid soils: Evaluation of soil fertility, nutrients pools and stoichiometry. Journal of Arid Environments, n. 166, p. 1-10, 2019. DOI: doi.org/10.1016/j.jaridenv.2019.03.006

PENDRILL, F.; PERSSON, U. M.; GODAR, J.; KASTER, T.; MORAN, D.; SCHMIDT, S.; WOOD, R. Agricultural and forestry trade drives large share of tropical deforestation emissions. Global Environmental Change, v. 56, p. 1-10, 2019. DOI: doi.org/10.1016/j.gloenvcha.2019.03.002.

ROBICHAUD, P. R.; JENNEWEIN, J.; SHARRATT. B. S.; LEWIS, S. A.; BROWN, R. $E$. Evaluating the effectiveness of agricultural mulches for reducing post-wildfire wind erosion. Aeolian Research, v. 27, p. 13-21, 2017. DOI: dx.doi.org/10.1016/j.aeolia.2017.05.001.

SILVA, G. F.; SANTOS, D.; SILVA, A. P.; SOUZA, J. M. Indicadores de qualidade do solo sob diferentes sistemas de uso na mesorregião do agreste paraibano. Revista Caatinga, v. $28, \quad$ n. 3 , p. $25-35,2015$. DOI: doi.org/10.1590/1983$21252015 v 28 n 303 r c$.

STAVI, I.; BARKAI, D.; KNOLL, Y. M.; ZAADY, E. Livestock grazing impact on soil wettability and erosion risk in post-fire agricultural lands. Science of the Total Environment, v. 573, p. 1203-1208, 2016. DOI: doi.org/10.1016/j.scitotenv.2016.03.126.

VIDAL, D. L.; SANTOS, D. P. A. Realidade territorial de unidades familiares no semiárido brasileiro. Tempo Social, v. 4, p. 1-6, 2016. DOI: doi.org/10.11606/01032070.ts.2016.105992.

VIGLIZZO, E. F.; RICARD, M. F.; TABOADA, M. A.; VÁZQUEZ-AMÁBILE, G. Reassessing the role of grazing lands in carbon-balance estimations: Meta-analysis and review. Science of the Total Environment, v. 661, p.531-542, 2019. DOI: doi.org/10.1016/j.scitotenv.2019.01.130.

ZERAATPISHEH, M.; BAKHSHANDEH, E.; HOSSEINI, M.; ALAVI, S. M. Assessig the effects of deforestation and intensive agriculture on the soil quality through digital soil mapping. Geoderma, v. 363, n. 114-139, 2020. DOI: doi.org/10.1016/j.geoderma.2019.114139. 